\title{
THICK FILM THERMAL PRINT HEADS
}

\author{
PETER BARNWELL \\ CorinTech Ltd., Ashford Mill, Station Road, Fordingbridge, Hampshire, England.
}

(Received April 10, 1979)

\begin{abstract}
Thermal print heads are finding widespread application in equipment as diverse as pocket calculators, chart recorders, and computer page printers. The use of thick film resistors to mark paper is one of the more common techniques and the design problems and performance available from such heads are the subject of this paper. Future developments of the technology are also discussed.
\end{abstract}

\section{INTRODUCTION}

In recent years the number of techniques for marking paper in chart recorders, printers and similar applications has increased dramatically. Until recently pen and ink systems were invariably used for recording graphics, and type and ribbon for recording alphanumerics. This is no longer the case.

One of the most significant newer approaches is that of thermal printing. An array of (or in more primitive cases a single) resistive element produces heat when driven from a suitable electrical signal. In turn a heat sensitive paper moving past the resistive element(s) is marked by the heat generated. A fast, quiet, reliable source of pattern recording thus results. Frequently the technology used for the manufacture of resistive elements, and the complex interconnection required, is a development of conventional thick film production.

This paper discusses the reasons behind this choice of technology, and its implementation. The problem associated with both the vital resistive element and the equally important connection system are described, and a number of design approaches are connsidered. Example of print heads produced by CorinTech Ltd. are used to illustrate the solutions adopted, and also the performance obtainable.

\section{PRINT TECHNIQUES}

\subsection{Why Thermal Printing?}

A wide variety of printing techniques are available, and each have their advantages and drawbacks. For production of graphics in chart recorders and similar instruments, the time honoured pen and ink system has the dual advantages of proven reliability (except for the actual inking system!) and apparently low cost. Unfortunately the problems associated with ink feed, mechanical delay and overshoot in the pen system, and the complexity of multi-channel recording count strongly against this system. Thermal printing overcomes these drawbacks by using a string of resistors across the width of the paper, the relevant resistor being addressed to produce a trace in the desired position. The obvious drawback of increased electronic complexity to access the head correctly is compensated by the reduced mechanical complexity, and becomes less severe with multi-channel operation where a time sharing system can be used.

Printers which are required to produce alphanumeric symbols already use a wide range of marking techniques. A variety of type and ribbon systems continues to be used, but they are both complex and more importantly noisy. Matrix impact printers are a logical development providing increased flexibility in print information, but are still relatively noisy and, due to the need to scan across the paper, relatively slow. Ink jet techniques are receiving great attention, and appear capable of achieving high performance in expensive systems such as computer print outs. Again the use of thermal printing overcomes a great deal of the objections, perhaps its most important characteristic in many applications being its silence.

For alpha-numeric printing a variety of techniques can be used:-

1) A small print head containing a 5 by 8 array (or similar) of resistors is scanned across the paper, printing each character as it moves. This is thermal equivalent to the matrix impact printer mentioned previously and has the same limitations with respect to speed. 
2) A seven segment pattern of resistors on the head is repeated by however many columns it is desired to print. The head now remains fixed and the paper is moved past the head in steps slightly greater than the character heights, printing an entire set of numbers at one time. Such a head in the form of $2+2$ digits for printing time is shown in Figure 4 . This technique has the advantage of high speed, at the cost of poor character quality and a restriction to number printing only.

3) An array of resistors can provide, if addressed correctly, a means of printing full alpha-numerics across the width of the paper with a fixed head. Characters are again printed in matrix form, and are of excellent quality even when small, and can be produced rapidly. Heads designed for producing graphics, as described previously can perfectly well be used for this purpose, thus giving a chart recorder the ability to print channel identification and scale information as well as producing traces. Alternatively heads can be designed in groups of resistors solely for the purpose of printing characters.

This paper will discuss solely the multiple dot approach to printing.

\subsection{The Choice of Technology}

Three basic technologies are in common use for thermal printing, and each has its own advantages and disadvantages. They may be summarised as follows:-

\subsubsection{Semiconductors. The use of silicon devices,} frequently in beam lead form, and normally mounted on a thick film substrate has been the approach of a number of companies, notably Texas Instruments. Heating is obtained by resistance within the doped semiconductor, and the diodes required for matrixing (see Section 4) are included automatically. In some cases the silicon chip is used as a switch, with the intention of simplifying drive circuitry.

Advantages of such a technique are the excellent wear characteristics of silicon, combined with the use of well established semiconductor manufacturing techniques for production of the print element.

Disadvantages however appear to be significant. There are severe constraints on the choice of dot size, complex power supplies are often required, and the price of such heads is too high for many applications. Additionally, as such a product represents a relatively low level of turnover by semiconductor house standards, supply of heads appears problematical.
2.2.2. Thin Film. An alternative approach, used mainly by Hewlett-Packard is to produce an array of thin-film resistors, and appropriate thin-film interconnections. The paper is now marked by the heat generated in the thin film resistors.

Advantages of this technique appear to be two fold. Firstly as thin-film circuits are commonly fabricated on glass, the poor thermal conductivity of the glass allows efficient heating of the resistor. Secondly, the inherent fine line definition of the thin-film process allows the production of high definition resistors.

Disadvantages are seen in a number of ways. The high cost of thin film circuit fabrication would appear to be a problem, although as print heads are a relatively high cost item this is not too important. Resistor robustness is inadequate to withstand the rubbing action of the paper moving past it, requiring a protective coating which slows down the print speed significantly.

2.2.3. Thick Film. Thick film technology can be used in essentially the same way as thin-film, to produce an array of resistors, and the interconnection pattern needed to address them.

Advantages are considerable; in particular the mechanical robustness of the thick film resistor under high pulsed power conditions allows very rapid marking of the paper. Additionally the ease of multilayering and the production of complex interconnections allows full matrixing of the resistor dots, and hence efficient use of connections to the print head. The ready bondability of thick film conductors allows the direct use of semiconductors on the substrate for decoding input information.

Disadvantages do again exist. The high thermal conductivity of the alumina normally used for substrates can require the use of high power levels. Additionally achieving high definition of the resistors is a problem. With careful design both these problems can be reduced dramatically as will be seen.

The flexibility of the thick film technique, combined with these advantages listed above have led to its use as the most common technology for print head production.

\section{THE PRINT HEAD RESISTOR}

It is convenient to consider the characteristics of the print head resistor in isolation from the connections. A number of requirements are placed on the resistor, many of which conflict with normal thick film 
requirements. These may be summarised:-

1) Low value (typically less than $100 \mathrm{ohms}$ ) to minimise drive voltage.

2) Smooth surface, and mechanical robustness

3) Screen printable to precise geometry

4) High pulse power handling

These requirements are met by careful choice of material and design, and no unique solution exists. A number of compromises have to be accepted, to provide the right answer for each application. The two areas of mechanical design and material aspects are now discussed:-

\subsection{Mechanical Design}

The majority of print head requirements require resistors of geometry well below the normal $1 \mathrm{~m} . \mathrm{m}$. dimensions used as a minimum for conventional circuits. For example CorinTech manufacture heads with a 0.2 pitch, requiring a $0.15 \mathrm{~m} . \mathrm{m}$. wide resistor.

Two solutions to this problem exist, both of which remove the need to print small resistors, placing the emphasis instead on high definition conductor printing. This is of course a much less severe problem, $0.1 \mathrm{~m} . \mathrm{m}$. lines and gaps being achievable with modern materials. The alternatives are illustrated in Figure 1, in each case the resistor being printed in a continuous strip across the full width of the head. The laser cut solution in Figure 1 (a) uses the conductor separation to define the resistor length, and laser cutting of the resistor to define width. Remarkably consistent resistors result from this approach, with a spread across a $64 \mathrm{~mm}$ head of typically $\pm 5 \%$ being experienced. Alternatively the conductor pattern can be used to determine the one dimension, and the resistor print the other dimensions as in Figure 1(b). This is economically attractive as it removes the expensive laser operation, but leads to less precise resistors, and if reduced to a fine pitch the presence of the conductor produces a significant dead zone between dots.

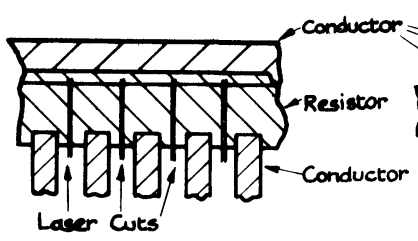

(a)

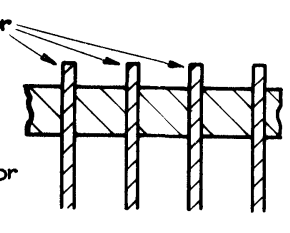

(b)
FIGURE 1 Definition of Resistor geometry.

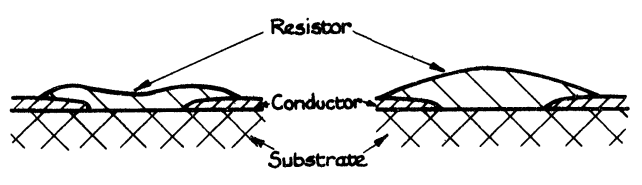

FIGURE 2 Resistor profile.

The author favours the approach of Figure 1(a) due to its generally better performance, and the heads described in Section 5 of this paper have all been made this way.

The other prime aspect of the resistor design is to do with its thickness, as it is vital that the paper is held in contact with the resistor, not the area surrounding it. This problem is illustrated in Figure 2. The normal thick film resistor profile provides very poor heat transfer to the paper, whereas if a thick resistor can be deposited as shown, greatly improved heat transfer results. Two techniques are combined to achieve this, firstly the gap between the conductors is filled in with a dielectric layer and secondly the resistor is printed very thickly. Hence a bump rather than a dip profile results.

\subsection{Material Choice}

Given that the resistor has been designed correctly as described above, then the thick film materials must be chosen carefully. The interaction between the conductor, the dielectric underlay and the resistor paste is a complex one. A satisfactory result can only be achieved by a prolonged evaluation of alternatives, and no general guidelines can be given. For example CorinTech use materials from several different manufacturers in achieving the performance required and a steady development has improved the marking speed capability of the dot by a factor of five times.

\section{THE INTERCONNECTION PROBLEM}

Given a correctly designed and made resistor, the rest of the work is concerned with getting connections to the resistors. Obviously if the head is made with individual leads to each resistor there is a severe connection problem due to the large number of dots. For example we have produced print heads with 512 dots, and a Japanese company is known to manufacture a head with 1024 dots on it.

As thick film is an ideal technology for producing multilayer interconnections, it makes sense to use this facility directly on the substrate. A matrix 


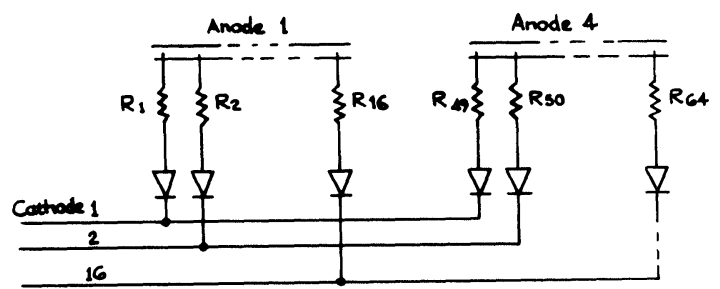

FIGURE 364 Resistor matrix.

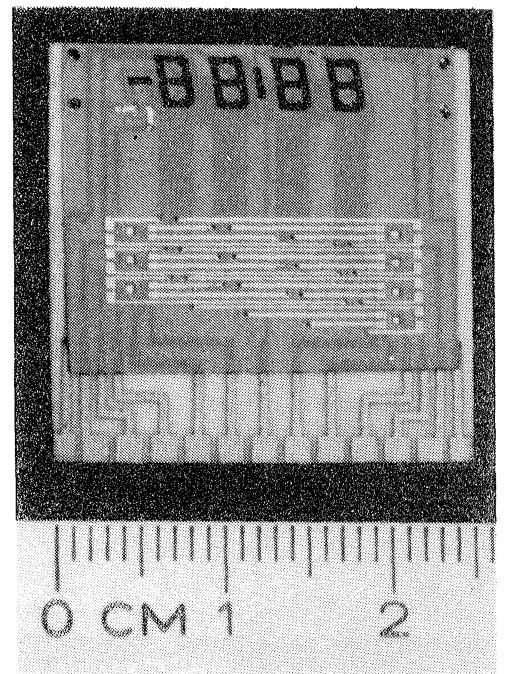

FIGURE 47 segment Thermal Printer.

connection pattern can be built, such as a $4 \times 16$ array to address 64 dots, as shown in Figure 3. Unfortunately due to the need to avoid parasitic conductor paths through the resistor, a diode has to be used with each resistor. However the use of multiple chip diodes allows these to be added efficiently and compactly. The detail design of such an interconnection system is complex if the most efficient use is to be made of the material, substrate area and production processing. As can be seen from the two heads illustrated in Figure 5, two totally different approaches have been used due to the differing specifications. They however all use a variation of the diode matrixing shown in Figure 3.

\section{PERFORMANCE OF TYPICAL HEADS}

Figure 5 shows 2 different print heads manufactured by CorinTech for widely differing applications.

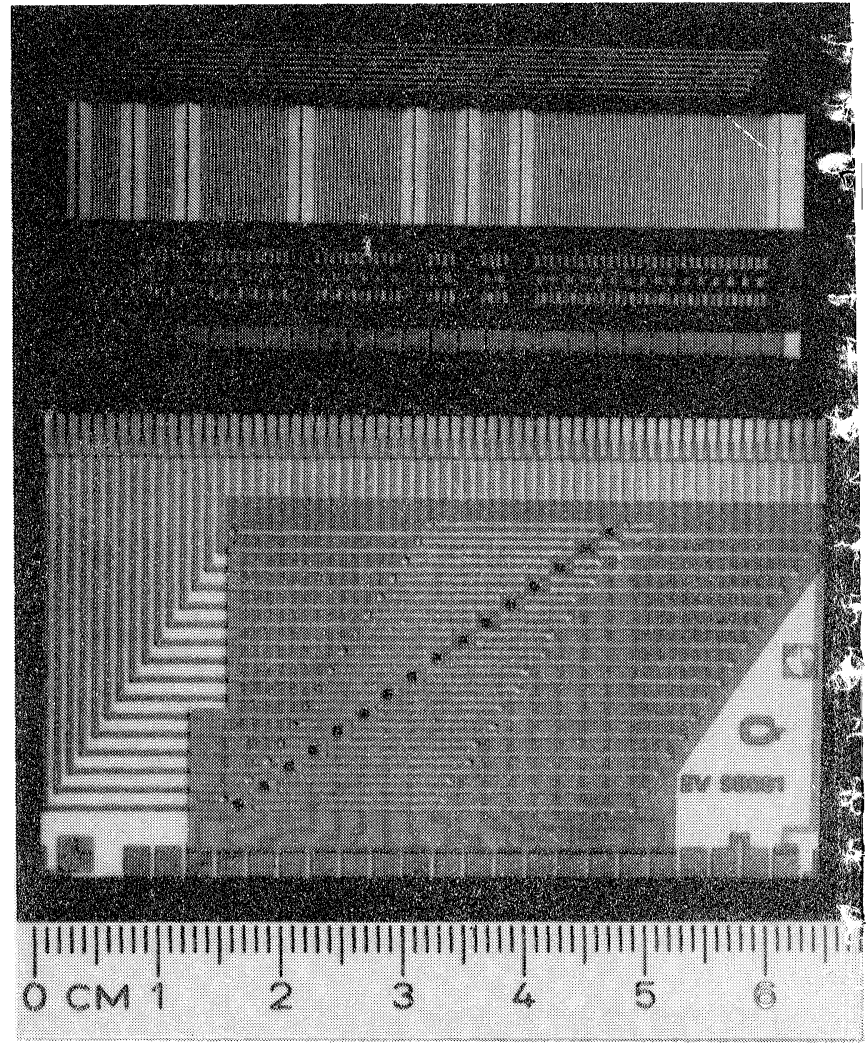

FIGURE 5 Line of dots Thermal Printer.

The larger head contains 64 resistors on a $1 \mathrm{~mm}$ pitch, and is designed to be edge stackable, hence four can be used to cover a $250 \mathrm{~mm}$ chart width. These heads have been developed for use in the Smiths Industries Servoscribe 700 recorder, which is the worlds first flat bed $250 \mathrm{~mm}$ thermal strip recorder. In a general purpose laboratory chart recorder of this type, response time of the head is of vital importance, as many applications require monitoring of fast transients. In order to provide a response time of $0.6 \mathrm{sec}$ across the full $250 \mathrm{~mm}$ width, with 3 channels, simple arithmetic shows that each dot must mark in $0.8 \mathrm{~ms}$ ! Careful development based on the principles already discussed have produced a head capable of meeting this highly demanding requirement, the problems being associated with the resistor design rather than the interconnection pattern. The dot pitch of $1 \mathrm{~mm}$ is of course simple to achieve, yet provides a $0.4 \%$ of full scale resolution which is highly acceptable for such a recorder.

The other head in Figure 5 has been developed for low speed high resolution printing, having 144 dots 
on a $0.3 \mathrm{~mm}$ pitch, grouped for both graphic and alpha-numeric printing. In this case the interconnections are the major design difficulty.

\section{FUTURE DEVELOPMENTS}

It has been shown that the use of thick film technology can provide a wide variety of types of thermal print heads. Future developments will be mainly concerned with the increased speed, higher dot density and larger heads. Additionally there will be the inevitable pressure on costs, in order to reduce print head prices. The key to most of these developments lies as ever in the availability of improved materials.

\subsection{Resistor Developments}

For print head use the resistor material must fulfil the following requirements:-

a) The ability to print and fire a thick print with a smooth surface, in order to allow optimum heat transfer into heat sensitive paper.

b) Rheology suited to high definition printing and firing, to remove as far as possible the need for laser machining.

c) Excellent pulse power handling, a criterion which does not necessarily coincide with good long term power handling.

Price of the material is relatively unimportant as the cost of the resistor is a small part of the total head cost.

The author knows of only one material specifically offered for thermal head use, and this unfortunately only offers the advantage of improved pulse power handling. Hence CorinTech have found it necessary to develop a specialised blend of materials to achieve desired performance.

\subsection{Interconnection Development}

The problem of interconnections to the resistor is, on occasion more complex than the actual resistor production. Whilst careful design and production can overcome the problems, the use of a gold conductor multilayer system is the standard.

Unfortunately the cost of a gold multilayer is extremely high, and frequently represents the largest single cost in head production. Several alternatives have been considered, but only one appears to possess the right mix of properties. This is to use a relatively high silver content platinum-silver material, with an appropriate dielectric. However in spite of testing a number of such systems, the problems associated with silver migration have been found to be severe, and gold has been retained for production.

This requirement for a low cost, high conductivity, reliable multilayer system is not unique to print heads and it is amazing that paste manufacturers do not appear prepared to offer such a system. Only one manufacturer has been found who lists a $\mathrm{Pt}-\mathrm{Ag} /$ Dielectric system for multilayer use, and these materials are not readily available in the U.K.

\subsection{The Substrate}

Little mention has so far been made of the effect of the substrate, as all the work described has been carried out on conventional $96 \%$ alumina substrates, with ground flat surface. Alumina is far from ideal as it is difficult to obtain in large sizes, and has a high thermal conductivity.

The ideal substrate is a low thermal conductivity material, obtainable in large flat sheets, and having high mechanical strength. Glass has been considered for this, as it fulfils two of the requirements, but its poor strength is a problem. Additionally, there has been a restricted choice of compatible thick film materials until recently. In the last 12 months the interest in porcelainised steel substrates has led to the announcement of several materials for use on glass. Future developments in this area would appear promising. 

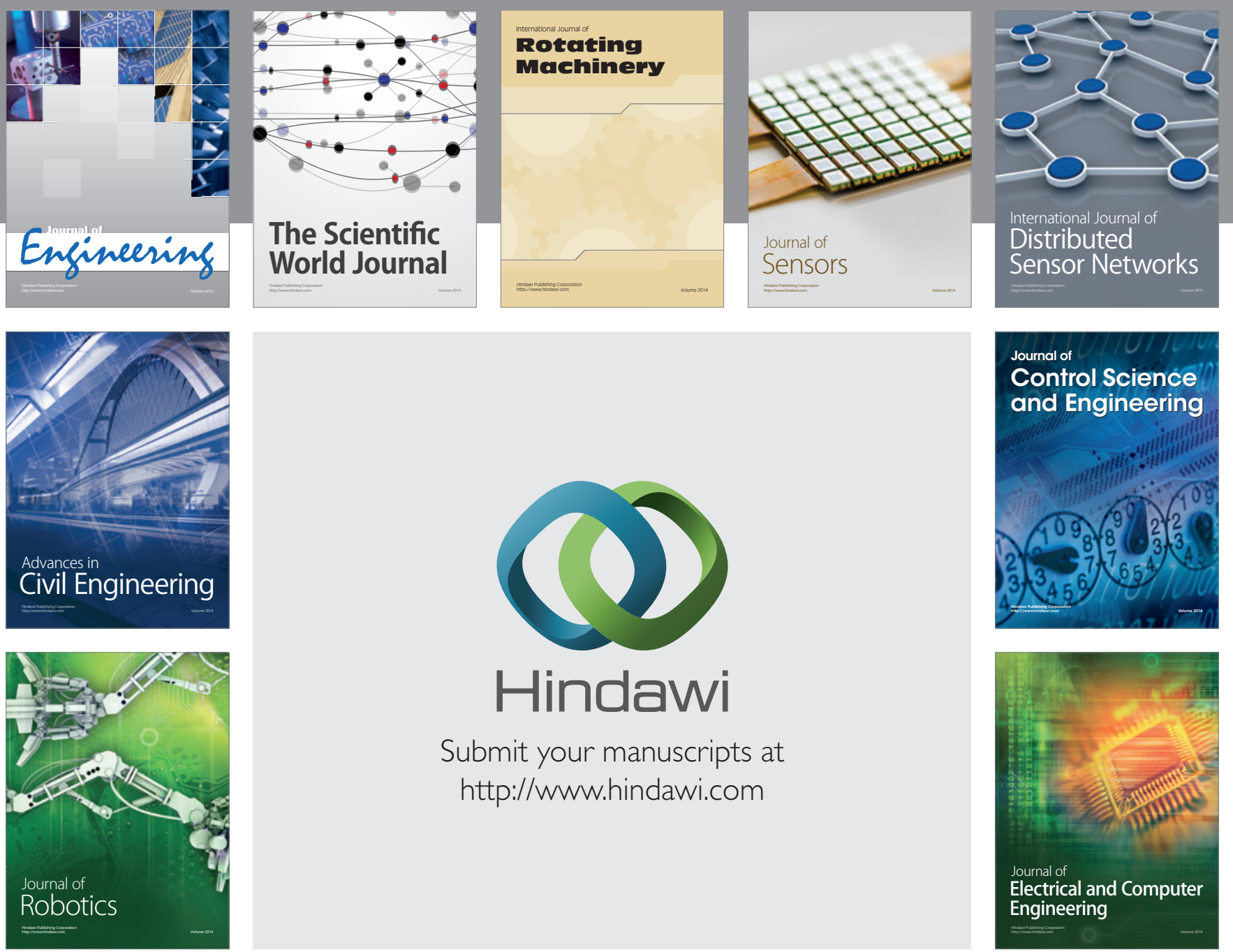

Submit your manuscripts at

http://www.hindawi.com
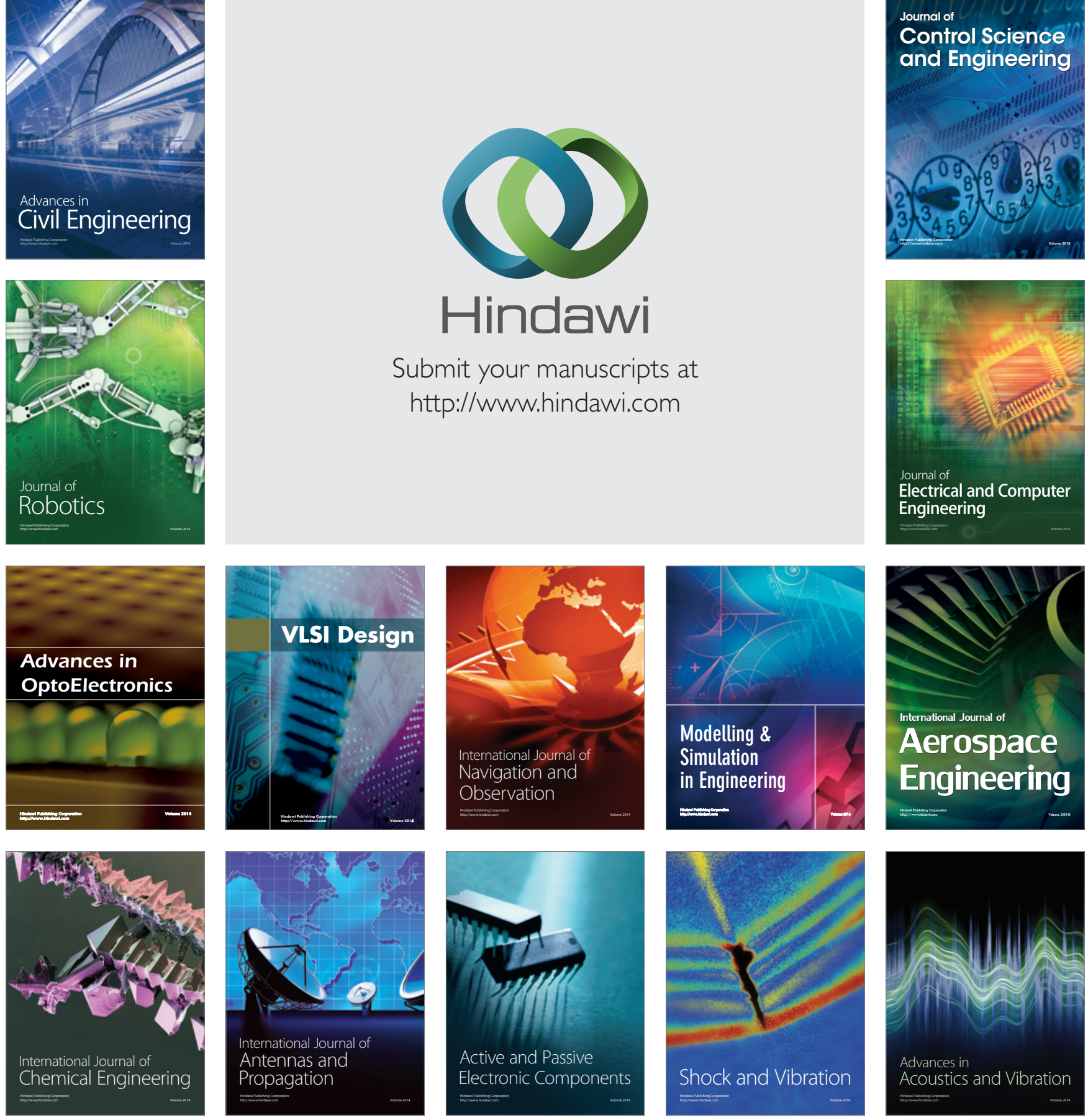